\title{
NONRANDON ANAPHASE SEGREGATION OF MITOTIC CHROMOSOMES
}

Sir:

When examining metaphase chromosomes, the early anaphase segregation of the chromatids of some chromosomes is sometimes observed. Since we had the feeling that chromatids of the E-group chromosomes separate earlier than those of other groups or pairs, we made an attempt to examine the distribution of eariy segregation by chromosome pairs or groups.

Blood cultures of 20 healthy adults (10 men and 10 women) were prepared according to the routine micromethod used in our laboratory (McCoy's 5A Medium, 72 hours culture time). The karyotype proved to be normal in each case. Altogether, 159 mitoses were photographed and set into karyotype. Segregation of the chromatids was scored when no connection between the sister chromatids was seen. Only cells in which 1,2 or 3 chromosomes displayed a segregation, were analysed. A total of 49 mitoses with 92 early segregations fulfilled these requirements.

Since no sex difference was found, the cumulative data on distribution of early segregation by chromosomes or groups are summarized in the Table.

Distribution of Early Anaphase Segregation by Chromosome Pairs or Groups

\begin{tabular}{lrrr}
\hline \hline $\begin{array}{c}\text { Chromosome } \\
\text { pair or } \\
\text { group }\end{array}$ & $\begin{array}{c}\text { Observed } \\
\text { frequency }\end{array}$ & $\begin{array}{c}\text { Expected } \\
\text { frequency }\end{array}$ & $\chi^{2}$ \\
\hline 1 & & 4 & 2.2 \\
2 & 13 & 4 & 20.2 \\
3 & 2 & 4 & 0.2 \\
$4-5$ & 7 & 8 & 0.1 \\
$6-X-12$ & 19 & 30 & 4.0 \\
$13-15$ & 1 & 12 & 10.1 \\
16 & 1 & 4 & 0.5 \\
17 & 6 & 4 & 1.0 \\
18 & 25 & 4 & 110.2 \\
$19-20$ & 6 & 8 & 0.5 \\
$21-22-Y$ & 12 & 10 & 0.4 \\
Total & 92 & 92 & 149.4 \\
& & DF $=10, \quad P<0.001$ \\
& & & \\
& & &
\end{tabular}

As shown by the figures, this distribution proved to be significantly nonrandom. The most conspicuous finding was the high frequency of early segregation in chromosomes No. 18, while Nos. 1, 13-15 and 16 appeared to be fairly stable in their morphology. The latter could be demonstrated in four cultures harvested after a longer colchicin treatment (5 instead of 2 hours). Here we found 18 cells in which all except one to three chromosomes had already been segregated, and No. 1 and the D-group were the commonest among the resistants. This phenomenon was also seen in 5 patients with Down's syndrome and in 3 infants with Patau's syndrome. However, no early segregation of chromosomes No. 18 was seen in 31 cells of three cases of trisomy 18 .

Because of the small number of observations it cannot be judged whether this nonrandomness is of any pathogenetic significance or not. It cannot be determined how much the phenomenon is influenced by technical factors either. However, since the nonrandom segregation proved to be fairly consequent in our material, and recently also Vig and Wodnicki (1974) reported on similar findings, it seems worthwile to investigate the problem on a large scale too.

Reference:

Vig B.K., Wodnicki J. 1974. Separation of sister centromeres in some chromosomes from cultured human leukocytes. J. Hered., 65: 149-152.

KÁROLY MÉHES

Department of Pediatrics

County Hospital

H-9002 Györ, Pf. 92, Hungary 